\title{
What Is the Frequency of Noise Generation in Modern Knee Arthroplasty and Is It Associated With Residual Symptoms?
}

\author{
Denis Nam MD, MSc, Toby Barrack BA, Ryan M. Nunley MD, \\ Robert L. Barrack MD
}

Published online: 13 January 2016

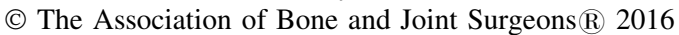

\begin{abstract}
Background Prior investigations have recognized the presence of patient-perceived noise generation after total knee arthroplasty (TKA). However, questions remain regarding its overall frequency after both TKA and unicompartmental knee arthroplasty (UKA) as well as with respect to its association with demographic and prosthesisrelated factors and its association with patient-reported outcomes.

Questions/purposes The purposes of this study were (1) to determine the frequency with which patients report noise coming from the knee after TKA or UKA; (2) to identify patient and prosthesis-related factors associated with noise generation; and (3) to ascertain whether noise coming from the knee is associated with residual symptoms after knee arthroplasty.

Methods A five-center survey study was designed to identify patient-perceived noise and to quantify the degree

Funding for this study was provided internally and with partial funding provided by Stryker Inc (Mahwah, NJ, USA) (RMN). All ICMJE Conflict of Interest Forms for authors and Clinical Orthopaedics and Related Research $\AA$ editors and board members are on file with the publication and can be viewed on request.

Clinical Orthopaedics and Related Research $₫$ neither advocates nor endorses the use of any treatment, drug, or device. Readers are encouraged to always seek additional information, including FDAapproval status, of any drug or device prior to clinical use. Each author certifies that his or her institution approved the human protocol for this investigation, that all investigations were conducted in conformity with ethical principles of research, and that informed consent for participation in the study was obtained.
\end{abstract}

D. Nam ( $₫)$, T. Barrack, R. M. Nunley, R. L. Barrack Department of Orthopaedic Surgery, Washington University School of Medicine/Barnes-Jewish Hospital, 660 S Euclid Avenue, Campus Box 8233, St Louis, MO 63110, USA e-mail: namd@wudosis.wustl.edu of residual symptoms and functional deficits in patients after TKA or UKA. Data were collected by an independent, third-party survey center, which administered questions about residual symptoms, function, and pre- and postoperative activity levels. Patients meeting prespecified inclusion criteria were specifically questioned regarding perceived noises from their knee within the last 30 days; those who reported hearing noises sometimes, often, or extremely often were categorized as positive. We retrospectively identified 2671 patients who underwent TKA and 744 patients who underwent UKA and who met inclusion criteria; the final survey population included 1580 patients who underwent TKA and 476 patients who underwent UKA (68\% response rate). TKA implant types included cruciate-retaining (59\%), posterior-stabilized (16\%), rotating-platform (13\%), gender-specific (7\%), and high-flex $(5 \%)$. Differences in baseline demographic variables were accounted for using multiple logistic regression statistical analyses. Chi square analyses were used to compare the frequency of residual symptoms in those patients with and without noise generation.

Results Overall, 27\% (557 of 2056; 95\% confidence interval [CI], 25-29) of all patients undergoing knee arthroplasty reported hearing grinding, popping, or clicking from their operative knee in the last 30 days. Men (odds ratio $[\mathrm{OR}], 1.3 ; 95 \% \mathrm{CI}, 1.0-1.6 ; \mathrm{p}=0.02)$ and younger patients $\left(\chi^{2}[\mathrm{df}=7]=67.3 ; \mathrm{p}<0.001\right)$ were more likely to report noise generation. After controlling for potential confounding variables, noise generation was more common after TKA $(29 \%)$ than UKA $(21 \%$; OR, 1.5 ; 95\% CI, $1.2-$ $2.0 ; \mathrm{p}<0.001)$. Among TKA designs, the likelihood of noise generation was greater in posterior-stabilized $(41 \%$; OR, 2.5; 95\% CI, 1.8-3.7; $\mathrm{p}<0.001)$, rotating-platform (45\%; OR, 2.8; 95\% CI, 1.9-4.2; p < 0.001), and genderspecific $(36 \% ;$ OR, 2.0; 95\% CI, 1.2-3.2; p $=0.007)$ 
designs than in cruciate-retaining (23\%) knees. Patientperceived noise generation was associated with residual symptoms, including difficulty getting in and out of a chair (38\% versus $25 \%, \mathrm{p}<0.001)$, limp (39\% versus $25 \%, \mathrm{p}<$ 0.001 ), swelling ( $42 \%$ versus $24 \%, \mathrm{p}<0.001$ ), and stiffness $(40 \%$ versus $23 \%, \mathrm{p}<0.001)$ compared with those who did not report noise generation after TKA.

Conclusions Patients frequently perceive noises coming from the knee after arthroplasty, more so in TKA than UKA. Patients reporting noises from the knee were more likely to report functional limitations and the presence of a limp, swelling, and stiffness. Surgeons should inform patients preoperatively of this possibility, because unmet patient expectations are known to negatively impact patient satisfaction after surgery. Subsequent investigations should focus on determining if there is a causal relationship between noise generation and residual symptoms after knee arthroplasty.

Level of Evidence Level III, therapeutic study.

\section{Introduction}

Noise generation after modern total joint arthroplasty has predominantly focused on ceramic-on-ceramic bearings in THA $[1,5,6,13,18,20,21,25]$. However, little information has been presented regarding the frequency of noise generation after TKA or unicompartmental knee arthroplasty (UKA). Greater than 25 years ago, patellar crepitation and "clunk" were described as an etiology of symptomatic noise generation after posterior-stabilized TKA $[9,10,24]$, yet with refinements in implant design and surgical technique, its frequency has decreased and it is now rarely discussed [12].

Despite advances in implant design and surgical technique, patients often report noise generation after TKA with the use of modern implant designs. In a series of 49 patients undergoing TKA, 69\% of patients reported noise production after their arthroplasties and also noted the magnitude of noise to worsen over time [19]. In addition, in a study of 465 TKAs, the incidence of noise generation was reported to vary widely based on implant design, but the authors did not correlate the impact of noise generation on patient-related outcome measures [16]. Furthermore, these prior studies have been limited by the potential for observer bias, the variability in which noise generation was assessed, and a lack of correlation with patient-reported outcomes. Furthermore, to our knowledge, no studies have specifically focused on the frequency of noise generation after UKA. Thus, questions remain regarding the true frequency of patient-perceived noise generation after modern knee arthroplasty along with its association with patientreported outcomes.

We therefore sought to determine the frequency, potential patient and prosthesis-related factors, and potential association of patient-perceived noise generation with residual symptoms after TKA and UKA with modern implant designs.

\section{Materials and Methods}

Five total joint centers (Washington University School of Medicine, St Louis, MO, USA; Rush University Medical Center, Chicago, IL, USA; Anderson Orthopaedic Clinic, Arlington, VA, USA; Joint Replacement Surgeons, Mooresville, IN, USA; Rothman Institute of Orthopaedics, Philadelphia, PA, USA) and an independent third party survey center (University of Wisconsin Survey Center [UWSC], Madison, WI, USA) participated. Three centers were academic practices, whereas two centers were private practices that provided training programs for orthopaedic residents and fellows. Each center had an active joint replacement registry, all surgeons were fellowship-trained performing more than 200 knee arthroplasties per year, and each contributed patients meeting the inclusion criteria. Each center had a particular interest and experience with a specific TKA component design and was beyond the learning curve associated with its use. Before initiation of the study, institutional review board approval was obtained at the Washington University School of Medicine to serve as the coordinating center, and each participating center obtained approval from its institutional review board.

Inclusion criteria for this study were (1) males or females at least 18 years of age and skeletally mature; (2) patients requiring primary knee surgery as a result of noninflammatory arthritis (degenerative joint disease) such as osteoarthritis or avascular necrosis; (3) and patients who had undergone a primary knee arthroplasty within 1 to 4 years before the start of the study and had a minimum of 1 year of clinical followup. Unfortunately, we are unable to comment on how many patients were excluded for having followup of less than 1 year because we can only present information on those patients who elected to participate in the study. We excluded (1) subjects with a history of infection or sepsis in the knee, fracture, dislocation, or revision to the operated knee; and (2) patients with extensive medical comorbidities including hypertension, renal failure, coronary artery disease, liver disease, sickle cell disease, respiratory disease, cancer, and other severe chronic conditions that have been expected to limit their activity level. These patients were excluded to compare well-performing implants. 
We retrospectively identified 2671 potential patients who underwent TKAs and 744 potential patients who underwent UKA and who met inclusion criteria. Of those, there were 325 TKAs (297 with TKAs performed less than 1 year or greater than 4 years before the initiation of the study, 18 whose surgical procedure was actually a revision of a prior prosthesis, six who had undergone a revision procedure after their index TKA, and four for extensive medical comorbidities) and 66 UKAs (50 performed less than 1 year or greater than 4 years before the initiation of the study, seven who received a lateral UKA or patellofemoral arthroplasty, six for extensive medical comorbidities, and three who had undergone a revision procedure after their index UKA) that were found to have exclusions during the screening section of the questionnaire, leaving 2346 eligible patients undergoing TKA and 678 eligible patients undergoing UKA. In addition, 341 individuals refused to participate, 224 were never available, 306 were not found as a result of a bad address/phone number, 39 had died, 18 did not complete the interview, and 40 had a language barrier. Overall, this left 1580 completed TKAs (1580 of 2346 eligible, a 67\% response rate) and 476 completed UKA (476 of 678 eligible, a 70\% response rate) interviews for final analysis (overall 68\% response rate). Patients undergoing TKA had a mean age of $60 \pm 8$ years at the time of surgery with $62 \%$ being women, and they were contacted at a mean of $3 \pm 1$ years postoperatively. Patients undergoing UKA had a mean age of $62 \pm 8$ years at the time of surgery with $53 \%$ being women, and they were contacted at a mean of $2 \pm 1$ years postoperatively (Table 1).

During this study interval, all centers were routinely using components of varying designs and manufacturers. TKA implant types included standard cruciate-retaining (59\%), posterior-stabilized fixed-bearing (16\%), rotatingplatform (13\%; of which $41 \%$ were cruciate-retaining and $59 \%$ were posterior-stabilized), gender-specific (7\%; all cruciate-retaining), and high-flex (5\%; all cruciate-retaining). UKA implant types included mobile-bearing (76\%) and fixed-bearing (24\%). Investigators queried their total joint registries and compiled a list of patients meeting the inclusion criteria. Each center was able to produce a list of patients who met the inclusion criteria including age, date of surgery, and complete contact information for each patient.

We used a previously described survey $[2,15]$ reporting specific data regarding function and residual symptoms 1 to 4 years after knee arthroplasty. The survey was designed by the UWSC in conjunction with Washington University and questions were adapted from recent investigations detailing residual symptoms and function after knee arthroplasty $[3$, $4,14]$. To eliminate observer bias, the UWSC, an independent, blinded third party, performed all data collection. The UWSC was selected for their expertise in collecting health data for state and federal agencies [7, 17] and for having no affiliation with any of the participating centers. Each center reviewed its joint registry to identify patients meeting eligibility criteria and provided the list to the coordinating center at Washington University where they were compiled into a master database. The implant details were removed and only the contact information, date, and side of surgery were provided to the UWSC to ensure anonymous, blinded administration of the survey. The coordinating center maintained a comprehensive list of implant details to decode by implant type after interviews were complete before data analysis. Patients received advance notification letters approximately 1 week before they were contacted by the survey center. Interviewers obtained verbal consent, a screening section ensured patients met the inclusion criteria, and the full questionnaire was administered to those patients who both provided verbal consent and were determined to be eligible and capable to participate. All interviews were conducted in English. The telephone survey protocol included 25 telephone call attempts per patient. The final data were sent from the UWSC through a secure website in SPSS format (Version 16.0; SPSS Inc, Chicago, IL, USA).

This report focused on the specific questions of the survey assessing noise generation (popping, clicking, or grinding) and their association with patient perceived limp,

Table 1. Demographics and postoperative UCLA activity scores

\begin{tabular}{llll}
\hline Variable & TKA & UKA & p value \\
\hline Number of patients & 1580 & 476 & $224(47 \%)$ \\
Male (number of patients) & $600(38 \%)$ & $252(53 \%)$ & $<0.001$ \\
Female (number of patients) & $980(62 \%)$ & $62(8)$ & $2(1)$ \\
Age at surgery (years)* & $60(8)$ & $7(2)$ & $<0.001$ \\
Followup (years)* & $3(1)$ & $7(2)$ & 0.001 \\
Postoperative UCLA score* & $7(2)$ \\
\hline
\end{tabular}

* Values are expressed as mean with the SD in parentheses; UKA = unicompartmental knee arthroplasty. 
stiffness, and function. Patients were queried about residual symptoms in their knee in the last 30 days before survey administration. Questions regarding symptoms and function had five categories as described by Likert [11]. The responses were grouped into two categories of "never/ rarely" and "sometimes/often/extremely often" for comparison during data analysis based on the methodology described by Bourne et al. [4].

We used descriptive statistics to present categorical data with frequency and percentage of patients reporting noise generation after TKA and UKA. Demographic and clinical variables such as age, gender, minority status ("minority" considered black, Hispanic, or nonwhite), education level, income level, length of followup, and UCLA activity scores were considered as potential confounders and were analyzed as potential patient-related factors associated with noise generation. Significant variables were then accounted for during multivariate analysis to examine the reporting of noise generation among implant designs in TKA and UKA. Chi square analyses and Fisher's exact tests were used to compare categorical data and to report the frequency of residual symptoms in those patients with and without noise generation. An independent statistician (MW) not involved in patient care performed all analyses using SAS 9.2 software (Cary, NC, USA). A p value $<0.05$ was considered statistically significant.

\section{Results}

Proportion of Patients Reporting Noises After Knee Arthroplasty

Overall, $27 \%$ (557 of 2056; 95\% confidence interval [CI], 25-29) of all patients undergoing knee arthroplasty reported hearing grinding, popping, or clicking from their operative knee "sometimes, often, or extremely often" in the last 30 days. After controlling for potential confounding variables, noise generation was more common after TKA (29\%) versus UKA (21\%; odds ratio [OR], 1.5 ; 95\% CI, $1.2-2.0 ; \mathrm{p}<0.001)$.

Association of Noises With Patient and Prosthesis Factors

Male gender (OR, 1.3; 95\% CI, 1.04-1.6; p = 0.02) and younger age category $\left(\chi^{2}[\mathrm{df}=7]=67.3 ; \mathrm{p}<0.001\right.$; Table 2) were associated with an increased reporting of noise generation. The frequency of noise generation was not related to minority status $(\mathrm{OR}, 0.8 ; 95 \% \mathrm{CI}, 0.5-1.4 ; \mathrm{p}$ $=0.4)$, education level $\left(\chi^{2}[\mathrm{df}=6]=9.2 ; \mathrm{p}=0.2\right.$; Table 3$)$, income level $\left(\chi^{2}[\mathrm{df}=4]=2.9 ; \mathrm{p}=0.6\right.$; Table 4$)$, or postoperative UCLA activity score $\left(\chi^{2}[\mathrm{df}=9]=13.8 ; \mathrm{p}=\right.$ 0.9; Table 5).

Among TKA designs, the likelihood of noise generation was greater in posterior-stabilized (41\%; OR, 2.5; $95 \% \mathrm{CI}$, 1.8-3.7; $\mathrm{p}<0.001)$, rotating-platform (45\%; OR, 2.8; $95 \%$ CI, 1.9-4.2; p < 0.001), gender-specific (36\%; OR, 2.0; 95\% CI, 1.2-3.2; p = 0.007), and high-flex (29\%; OR, 1.2; $95 \% \mathrm{CI}, 1.0-1.4 ; \mathrm{p}=0.005)$ designs versus the standard cruciate-retaining $(23 \%)$ design. When analyzing only women undergoing TKA, those with a gender-specific design were more likely to report noise generation versus those with a standard cruciate-retaining TKA (OR, 1.9; 95\% CI, 1.2-3.1; $\mathrm{p}=0.009)$. When comparing noise generation in patients with the same cruciate-retaining TKA, there was no difference in noise generation between those who did or did not have a patella resurfacing (OR, 1.1; 95\% CI, 0.7-1.9; $\mathrm{p}=0.6$ ).

Noises From the Knee and Residual Knee Symptoms

Patient-perceived noise generation was associated with an increased reporting of residual symptoms after both TKA and UKA. Patients in the TKA cohort who reported noise generation were more likely to report difficulties getting in and out of a chair, the presence of a limp, swelling, and stiffness (Table 6). Of the 536 patients who reported stiffness in their knee, $40 \%$ (214 of 536) noted the presence of noise generation versus $23 \%$ (241 of 1042) of those who rarely reported stiffness $(\mathrm{OR}, 2.2 ; 95 \% \mathrm{CI}, 1.8-2.8 ; \mathrm{p}<$ 0.001).

Among patients in the UKA cohort, no difference was present among mobile- versus fixed-bearing designs for noise generation (OR, 1.3; 95\% CI, 0.8-2.1; p = 0.4). Patients in the UKA cohort who reported noise generation were more likely to report difficulties getting in and out of a car, the presence of a limp, swelling, and stiffness (Table 7). Of the 142 patients who reported stiffness in their knee, 39\% (55 of 142) noted the presence of noise generation versus $13 \%$ (44 of 233) of those who rarely reported stiffness (OR, 4.1; 95\% CI, 2.6-6.6; $\mathrm{p}<0.001)$.

\section{Discussion}

Patient-perceived noise generation is a recognized symptom after TKA, yet prior reports have been limited by their small cohort sizes and failure to determine its potential association with patient-reported outcomes [12, 16]. Prior investigations have also been limited by the potential for observer bias diminishing both the true frequency and potential impact of noise generation on patient function. Therefore, this study's purpose was to determine the 
Table 2. Noise generation based on age category

\begin{tabular}{|c|c|c|c|}
\hline Age category (years) & Never/rarely hears noise & Sometimes/often/extremely often hears noise & Total \\
\hline $18-29$ & $1(50 \%)$ & $1(50 \%)$ & 2 \\
\hline $30-39$ & $3(30 \%)$ & $7(70 \%)$ & 10 \\
\hline $40-49$ & $73(53 \%)$ & $64(47 \%)$ & 137 \\
\hline $50-55$ & $214(64 \%)$ & $123(37 \%)$ & 337 \\
\hline $56-60$ & $313(70 \%)$ & $136(30 \%)$ & 449 \\
\hline $61-65$ & $172(81 \%)$ & $41(19 \%)$ & 213 \\
\hline $66-70$ & $197(80 \%)$ & $50(20 \%)$ & 247 \\
\hline $71-80$ & $150(81 \%)$ & $35(19 \%)$ & 185 \\
\hline
\end{tabular}

Table 3. Noise generation based on highest level of education each patient completed

\begin{tabular}{|c|c|c|c|}
\hline Education level & Never/rarely hears noise & Sometimes/often/extremely often hears noise & Total \\
\hline Never attended school & $1(100 \%)$ & $0(0 \%)$ & 1 \\
\hline Grades $1-8$ & $8(89 \%)$ & $1(11 \%)$ & 9 \\
\hline Grades 9-11 & $25(70 \%)$ & $11(31 \%)$ & 36 \\
\hline Grade 12 or GED (graduate) & $300(67 \%)$ & $146(33 \%)$ & 446 \\
\hline College 1 year to 3 years & $340(74 \%)$ & $117(26 \%)$ & 457 \\
\hline College 4 years or more (graduate) & $221(69 \%)$ & $100(31 \%)$ & 321 \\
\hline Postgraduate 1 year or more & $223(74 \%)$ & $80(27 \%)$ & 303 \\
\hline
\end{tabular}

Table 4. Noise generation based on annual household income level

\begin{tabular}{|c|c|c|c|}
\hline Income level (USD) & Never/rarely hears noise & Sometimes/often/extremely often hears noise & Total \\
\hline Less than 25,000 & $126(74 \%)$ & $44(26 \%)$ & 170 \\
\hline 25,000 to less than 50,000 & $192(74 \%)$ & $66(26 \%)$ & 258 \\
\hline 50,000 to less than 75,000 & $201(69 \%)$ & $89(31 \%)$ & 290 \\
\hline 75,000 to less than 100,000 & $168(70 \%)$ & $71(30 \%)$ & 239 \\
\hline 100,000 or more & $314(70 \%)$ & $134(30 \%)$ & 448 \\
\hline
\end{tabular}

Table 5. Noise generation based on UCLA activity score

\begin{tabular}{|c|c|c|c|}
\hline UCLA activity score & Never/rarely hears noise & Sometimes/often/extremely often hears noise & Total \\
\hline 1 & $0(0 \%)$ & $1(100 \%)$ & 1 \\
\hline 2 & $5(63 \%)$ & $3(38 \%)$ & 8 \\
\hline 3 & $14(63 \%)$ & $8(36 \%)$ & 22 \\
\hline 4 & $46(74 \%)$ & $16(26 \%)$ & 62 \\
\hline 5 & $56(81 \%)$ & $13(19 \%)$ & 69 \\
\hline 6 & $366(76 \%)$ & $115(24 \%)$ & 481 \\
\hline 7 & $108(67 \%)$ & $53(33 \%)$ & 161 \\
\hline 8 & $142(75 \%)$ & $48(25 \%)$ & 190 \\
\hline 9 & $109(69 \%)$ & $50(31 \%)$ & 159 \\
\hline 10 & $203(66 \%)$ & $105(34 \%)$ & 308 \\
\hline
\end{tabular}


Table 6. Noise generation and residual symptoms in patients undergoing TKA

\begin{tabular}{lcc}
\hline Residual symptom & Never/rarely hears noise & $\begin{array}{l}\text { Sometimes/often/ } \\
\text { extremely often hears noise }\end{array}$ \\
\hline Do you have problems getting in and out of a car? & $773(65 \%)$ & $422(35 \%)$ \\
Never/rarely & $348(63 \%)$ & $205(37 \%)$ \\
Sometimes/often/extremely often & $849(75 \%)$ & $284(25 \%)$ \\
Do you have problems getting in and out of a chair? & $170(38 \%)$ \\
Never/rarely & $273(62 \%)$ & \\
Sometimes/often/extremely often & $841(75 \%)$ & $280(25 \%)$ \\
In the last 30 days, how often do you limp while walking? & $176(39 \%)$ \\
Never/rarely & $275(61 \%)$ & $284(24 \%)$ \\
Sometimes/very often/extremely often & $883(76 \%)$ & $173(42 \%)$ \\
In the last 30 days, how often did you experience swelling? & & \\
Never/rarely & $239(58 \%)$ & $241(23 \%)$ \\
Sometimes/very often/extremely often & $801(77 \%)$ & $214(40 \%)$ \\
In the last 30 days, how often did you experience stiffness? & $322(60 \%)$ & $<0.001$ \\
Never/rarely & $<0.001$ \\
Sometimes/very often/extremely often &
\end{tabular}

Table 7. Noise generation and residual symptoms in patients undergoing unicompartmental knee arthroplasty

\begin{tabular}{lcc}
\hline Residual symptom & Never/rarely hears noise & $\begin{array}{l}\text { Sometimes/often/ } \\
\text { extremely often hears noise }\end{array}$ \\
\hline Do you have problems getting in and out of a car? & $302(83 \%)$ & $61(17 \%)$ \\
Never/rarely & $72(66 \%)$ & $37(34 \%)$ \\
Sometimes/often/extremely often & $303(81 \%)$ & $72(19 \%)$ \\
Do you have problems getting in and out of a chair? & $73(73 \%)$ & $27(27 \%)$ \\
Never/rarely & $300(84 \%)$ & $57(16 \%)$ \\
Sometimes/often/extremely often & $73(64 \%)$ & $41(36 \%)$ \\
In the last 30 days, how often do you limp while walking? & \\
Never/rarely & $316(83 \%)$ & $64(17 \%)$ \\
Sometimes/very often/extremely often & $58(62 \%)$ & $35(38 \%)$ \\
In the last 30 days, how often did you experience swelling? & \\
Never/rarely & $289(87 \%)$ & $44(13 \%)$ \\
Sometimes/very often/extremely often & $87(61 \%)$ & $55(39 \%)$ \\
In the last 30 days, how often did you experience stiffness? & $<0.001$ \\
Never/rarely & & \\
Sometimes/very often/extremely often &
\end{tabular}

frequency, potential patient and prosthesis-related factors, and potential association of patient-perceived noise generation with residual symptoms after TKA and UKA with modern implant designs. We found that $27 \%$ of patients undergoing knee arthroplasty report noise generation postoperatively, which was more common after TKA versus UKA. Furthermore, the presence of noise generation was associated with an increased frequency of residual symptoms and limitations in functional activities after knee arthroplasty regardless of whether a TKA or UKA was performed.

This study had several limitations that must be recognized before interpretation of our data. First, given the retrospective nature of this study, we can only determine the presence of an association of noise generation with residual symptoms. Thus, we recognize that noise generation itself might not be the cause of limitations in patient function. Second, use of a retrospective survey method has 
the potential for recall bias, but we tried to limit the degree of bias by only including patients who received a knee arthroplasty within 1 to 4 years of the start of the study. However, because we only included patients with 1-year followup, it is possible that patients who do not have 1-year followup (and thus were excluded) or who had undergone revision surgery may experience increased noise generation than we have reported. Thus, this study may represent a best-case analysis in terms the frequency of noise generation, residual symptoms, and functional limitations. Third, although the survey instrument has previously been reported and is adapted from commonly used orthopaedic scores, it has not been validated in terms of its repeatability, ceiling effects, and other important parameters. Fourth, it is difficult to determine the exact etiology of noise generation and whether this is truly implant-related or originates from the soft tissue. However, because the frequency of noise generation in those receiving a cruciateretaining implant was significantly less than with all other designs, this strongly implies the bearing surface is the culprit of noise generation in most cases. Lastly, although this study possessed a large sample size, only $68 \%$ of eligible patients completed the survey. Thus, it is possible that our sample may misrepresent those who elected not to participate [8]. Unfortunately, we are not able to comment on the demographics and activity level of those patients who refused to participate because only patients participating in the survey were considered to have consented to the study and have their information collected; institutional review board restrictions precluded data collection and reporting on patients who did not elect to participate in the telephone survey, because part of the survey was an implied consent to participate in the study.

Approximately $30 \%$ of patients who underwent TKA in this study reported the presence of noise generation "sometimes, often, or extremely often." This frequency is much lower than that previously reported, because up to $69 \%$ of patient undergoing TKA have been shown to report noise generation [19]. However, this prior study only included 49 patient responses versus close to 1600 TKA responses in the current investigation. Furthermore, as a result of the size of their cohort, potential predictive factors of noise generation after TKA were unable to be assessed. Reporting of noise generation was not related to minority status, education level, income level, or postoperative UCLA activity score in our study, although male gender and younger age were associated with an increased reporting of noise generation. We theorize that although not captured by the UCLA activity score, younger male patients may have a tendency to be more demanding on their arthroplasties, perhaps contributing to their increased reporting of noise generation. To our knowledge, this is the first study to report demographic-related factors that may increase the perception of noise generation after knee arthroplasty.

Patients receiving a posterior-stabilized TKA design had the greatest likelihood of reporting noise, whereas patients receiving a cruciate-retaining design were least likely to report noise. The impact of prosthesis design on noise generation has previously been reported because noise-related symptoms were present in $12 \%$ of patients with a medial pivot, $4 \%$ with anterior and posterior cruciate ligament-retaining, $31 \%$ with a posterior cruciate-retaining, $33 \%$ with a posterior-stabilized, and $42 \%$ with a mobilebearing TKA design [16]. This study demonstrated similar findings for the cruciate-retaining (23\%) and posteriorstabilized $(41 \%)$ designs, perhaps suggesting that noise generation is more common with certain implant designs after TKA.

Noise generation was found to be of potential clinical importance in patients after both TKA and UKA, because it was associated with increased stiffness and swelling. A prior study by Lonner et al. [12] noted implant clicking and grinding to be prodromal symptoms related to intraoperative findings including polyethylene wear, osteolysis, synovitis, and component breakage. However, noise generation is possible from all moving joints with proposed etiologies being release of gas from synovial fluid during joint separation causing cracking [22], snapping from tendons or soft tissues moving over bony or implant prominences, and squeaking from edge loading of hard surfaces or potential impingement [23]. Thus, although this study cannot determine the source of noise generation, it remains critical to note that noise generation has a potential association with residual symptoms and functional limitations after knee arthroplasty.

This study found a high frequency of patient-perceived noise generation after knee arthroplasty, which was more common after TKA versus UKA. Patients reporting noise generation after knee arthroplasty were more likely to report functional limitations and the presence of a limp, swelling, and stiffness. Bourne et al. [4] found that among patients who were dissatisfied after TKA, 49\% stated that their expectations had not been met. It is reasonable to assume patients, unless educated preoperatively, will expect a TKA to be quiet and fail to produce noise. Therefore, it is critical that surgeons inform patients preoperatively of the possibility of noise generation after knee arthroplasty. It remains unclear if a clear source of noise generation after knee arthroplasty exists or if this is multifactorial. Future investigations should focus on determining if a causal relationship exists between patientreported outcomes and residual symptoms. Furthermore, randomized controlled studies may further elucidate whether specific implant designs used in TKA and UKA increase the likelihood of noise generation and residual 
symptoms. Subsequent investigations should focus on modifications in surgical technique or implant designs that decrease the frequency of noise generation after knee arthroplasty.

Acknowledgments We thank Dr Javad Parvizi, Dr William G. Hamilton, Dr Douglas A. Dennis, Dr Michael E. Berend, Dr Keith R. Berend, Dr Adolph V. Lombardi, Dr Craig J. Della Valle, and Dr Michael Wallendorf for their contributions to this work.

\section{References}

1. Amanatullah DF, Landa J, Strauss EJ, Garino JP, Kim SH, Di Cesare PE. Comparison of surgical outcomes and implant wear between ceramic-ceramic and ceramic-polyethylene articulations in total hip arthroplasty. J Arthroplasty. 2011;6(Suppl):72-77.

2. Barrack RL, Ruh EL, Berend ME, Della Valle CJ, Engh CA Jr, Parvizi J, Clohisy JC, Nunley RM. Do young, active patients perceive advantages after surface replacement compared to cementless total hip arthroplasty? Clin Orthop Relat Res. 2013;471:3803-3813.

3. Barrack RL, Ruh EL, Chen J, Lombardi AV,Jr, Berend KR, Parvizi J, Della Valle CJ, Hamilton WG, Nunley RM. Impact of socioeconomic factors on outcome of total knee arthroplasty. Clin Orthop Relat Res. 2014;472:86-97.

4. Bourne RB, Chesworth BM, Davis AM, Mahomed NN, Charron KD. Patient satisfaction after total knee arthroplasty: who is satisfied and who is not? Clin Orthop Relat Res. 2010;468:57-63.

5. 5. Brockett CL, Williams S, Jin Z, Isaac GH, Fisher J. Squeaking hip arthroplasties: a tribological phenomenon. J Arthroplasty. 2013;1:90-97.

6. Buttaro MA, Zanotti G, Comba FM, Piccaluga F. Squeaking in a delta ceramic-on-ceramic uncemented total hip arthroplasty.J Arthroplasty. 2012;6:1257-1259.

7. Centers for Disease Control and Prevention (CDC). Prevalence of cholesterol screening and high blood cholesterol among adultsUnited States, 2005, 2007, and 2009. MMWR Morb Mortal Wkly Rep. 2012;61:697-702.

8. Fincham JE. Response rates and responsiveness for surveys, standards, and the journal. Am J Pharm Educ. 2008;2:43.

9. Hozack WJ, Rothman RH, Booth RE Jr, Balderston RA. The patellar clunk syndrome. A complication of posterior stabilized total knee arthroplasty. Clin Orthop Relat Res. 1989;241:203208.
10. Ip D, Ko PS, Lee OB, Wu WC, Lam JJ. Natural history and pathogenesis of the patella clunk syndrome. Arch Orthop Trauma Surg. 2004;9:597-602.

11. Likert R. A technique for the measurement of attitudes. Arch.Psychol. 1932:1-55.

12. Lonner JH, Jasko JG, Bezwada HP, Nazarian DG, Booth RE Jr. Incidence of patellar clunk with a modern posterior-stabilized knee design. Am J Orthop (Belle Mead NJ). 2007;10:550-553.

13. Mai K, Verioti C, Ezzet KA, Copp SN, Walker RH, Colwell CW Jr. Incidence of 'squeaking' after ceramic-on-ceramic total hip arthroplasty. Clin Orthop Relat Res. 2010;468:413-417.

14. Noble PC, Conditt MA, Cook KF, Mathis KB. The John Insall Award: Patient expectations affect satisfaction with total knee arthroplasty. Clin Orthop Relat Res. 2006;452:35-43.

15. Nunley RM, Nam D, Berend KR, Lombardi AV, Dennis DA, Della Valle CJ, Barrack RL. New total knee arthroplasty designs: do young patients notice? Clin Orthop Relat Res. 2015;473:101-108.

16. Pritchett JW. A comparison of the noise generated from different types of knee prostheses. J Knee Surg. 2013;2:101-104.

17. Radler BT, Ryff CD. Who participates? Accounting for longitudinal retention in the MIDUS national study of health and wellbeing. J Aging Health. 2010;3:307-331.

18. Sariali E, Klouche S, Mamoudy P. Ceramic-on-ceramic total hip arthroplasty: is squeaking related to an inaccurate three-dimensional hip anatomy reconstruction? Orthop Traumatol Surg Res. 2014;4:437-440.

19. Sharkey PF, Miller AJ. Noise, numbness, and kneeling difficulties after total knee arthroplasty: is the outcome affected? $J$ Arthroplasty. 2011;8:1427-1431.

20. Stanat SJ, Capozzi JD. Squeaking in third- and fourth-generation ceramic-on-ceramic total hip arthroplasty: meta-analysis and systematic review. J Arthroplasty. 2012;3:445-453.

21. Tai SM, Munir S, Walter WL, Pearce SJ, Walter WK, Zicat BA. Squeaking in large diameter ceramic-on-ceramic bearings in total hip arthroplasty. J Arthroplasty. 2015;2:282-285.

22. Unsworth A, Dowson D, Wright V. 'Cracking joints.' A bioengineering study of cavitation in the metacarpophalangeal joint. Ann Rheum Dis. 1971;4:348-358.

23. Walter WL, Yeung E, Esposito C. A review of squeaking hips. $J$ Am Acad Orthop Surg. 2010;6:319-326.

24. Wong JW, Yau PW, Chiu PK. Arthroscopic treatment of patellar symptoms in posterior stabilized total knee replacement. Int Orthop. 2002;4:250-252.

25. 25. Wyatt MC, Jesani S, Frampton C, Devane P, Horne JG. Noise from total hip replacements: a case-controlled study. Bone Joint Res. 2014;6:183-186. 